\title{
Rectangling Stereographic Projection for Wide-Angle Image Visualization
}

\author{
Che-Han Chang ${ }^{1}$ Min-Chun $\mathrm{Hu}^{2} \quad$ Wen-Huang Cheng ${ }^{3} \quad$ Yung-Yu Chuang ${ }^{1 *}$ \\ ${ }^{1}$ National Taiwan University $\quad{ }^{2}$ National Cheng Kung University ${ }^{3}$ Academia Sinica
}

\begin{abstract}
This paper proposes a new projection model for mapping a hemisphere to a plane. Such a model can be useful for viewing wide-angle images. Our model consists of two steps. In the first step, the hemisphere is projected onto a swung surface constructed by a circular profile and a rounded rectangular trajectory. The second step maps the projected image on the swung surface onto the image plane through the perspective projection. We also propose a method for automatically determining proper parameters for the projection model based on image content. The proposed model has several advantages. It is simple, efficient and easy to control. Most importantly, it makes a better compromise between distortion minimization and line preserving than popular projection models, such as stereographic and Pannini projections. Experiments and analysis demonstrate the effectiveness of our model.
\end{abstract}

\section{Introduction}

Capturing a scene with a wide field of view from a single viewpoint records rich visual information of the scene. Such wide-angle images (full spherical panoramas at the extreme) can be obtained from stitching multiples images taken from the same viewpoint, or from shooting with fisheye lens. The recorded information can be defined with a viewing sphere which stores the incident radiance at the viewpoint from any incoming direction. For viewing wide-angle images defined on a viewing sphere, it is often required to map from the viewing sphere to an image plane. However, it is impossible to map from a sphere to a plane without introducing distortions. Thus, most projection models trade off different types of distortions and none can avoid all distortions. Also, different applications could have different requirements and desired properties. For our applications, viewing wide-angle perspective images, they include the following. (1) Minimal distortion: the shape should be preserved locally so that the image content is not severely distorted. Otherwise, the image will look stretched

\footnotetext{
*This work was partly supported by grants NSC101-2628-E-002-031MY3 and NSC102-2622-E-002-013-CC2.
}

or compressed. (2) Line preserving: scene lines should remain straight in the projection because human is often more sensitive to distortions of straight lines. Vertical/horizontal lines and lines passing through vanishing points are especially important. (3) Rectangular outline: it is preferred that the projected images have rectangular boundaries.

None of existing projection models can reach all these goals. Figure 1 demonstrates several popular projection models. In this example, the scene exhibits perspective effects with a vanishing point at the center of the image. Rectilinear projection (or gnomonic projection) preserves most scene lines, but exhibits extensive stretches for a large FOV. In addition, when the FOV approaches the theoretical limit (180 degrees), it requires infinite space for displaying the image. Cylindrical projections (equirectangular and Mercator) preserve the straightness of vertical scene lines. However, they reduce the perspective effects because scene lines towards the vanishing point become curved. Additionally, equirectangular projection severely distorts the area near the zenith and nadir. To preserve conformality, Mercator projection requires infinite space to show the whole view along the vertical direction. Thus, areas near the zenith and the nadir have to be cropped. Stereographic projection performs well on minimizing distortions, but several prominent lines become curved in the projection and the resultant image is not rectangular. Pannini projection, on the other hand, produces a rectangular image, and preserves vertical lines and scene lines towards the vanishing point. But it does not preserve horizontal lines and generates severe distortions around the zenith and nadir. Additionally, cropping is required because of its ineffective vertical FOV.

This paper proposes a projection model which strikes a good compromise among these desired properties. As shown in Figure 1, similar to stereographic projection, our model has a good conformality property and less content distortions. It further makes the outline of the image more rectangular. In addition, it preserves vertical scene lines and scene lines passing through the vanishing point in the same way Pannini projection does. Finally, it also better preserves horizontal lines that Pannini projection doesn't. It is particularly evident from the signs hung above the doors at the center. 


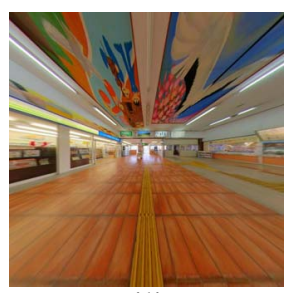

Rectilinear

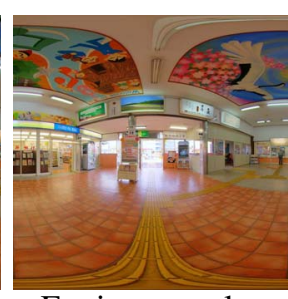

Equirectangular

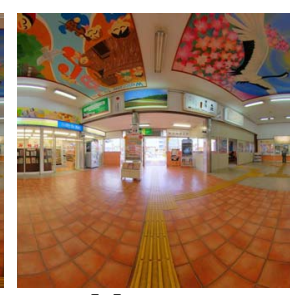

Mercator

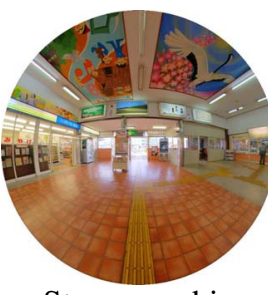

Stereographic

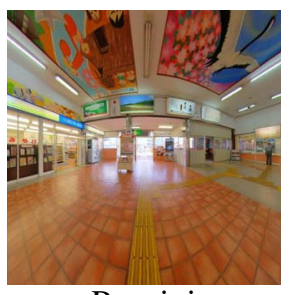

Pannini

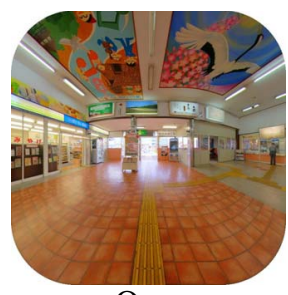

Ours

Figure 1: Comparisons of the proposed projection model with several popular projection models.

Our model consists of two steps. The first step projects the viewing hemisphere onto a swung surface. The second step maps the projection on the swung surface onto the image plane through the perspective projection. The key is the design of the swung surface. We construct our swung surface by rotating and scaling a circular profile along a rounded rectangular trajectory. We show that the proposed model unifies several projection models. We also demonstrate the model on applications of viewing wide-angle images and creating thumbnails for full spherical panoramas.

\section{Related work}

For panoramic projections, Zelnik-Manor et al. [10] proposed a multi-plane projection as an alternative to cylindrical projection. The cylindrical surface is replaced by several planes. Thus, the resulting panorama is composed by several perspective projections with the same viewpoint but with different projection planes. Kopf et al. [4] proposed an interactive viewer for gigapixel panoramas. They employed an adaptive projection which allows for smooth transition between the rectilinear and cylindrical projections.

For wide-angle images, Zorin and Barr [11] proposed a one-parameter family of projections that interpolate between the rectilinear and stereographic projections. Ying and $\mathrm{Hu}$ [9] proposed a unified imaging model for central catadioptric and fisheye cameras. Sharpless et al. [6] proposed Pannini projection for viewing wide-angle perspective images. Carroll et al. [2] presented a content-based approach that finds an optimal mapping function according to user-drawn lines. Based on this approach, Wei et al. [8] proposed a fisheye video correction method. Both the method of Carroll et al. [2] and our method can be used for viewing wide-angle images. However, their method requires users to draw lines and our method can be fully automatic.

There exist researches on creating panoramas from different viewpoints. Agarwala et al. [1] proposed a system for creating multi-viewpoint panoramas of street scenes from fisheye videos. Kopf et al. [3] presented a system for browsing multi-perspective street views. Our projection model is designed for the single-viewpoint configuration, different from these approaches.

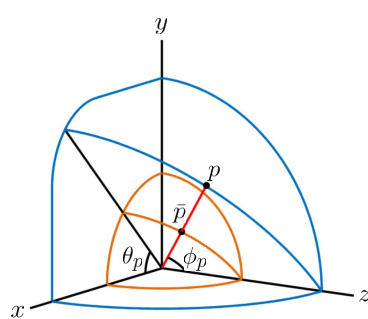

(a) The first step

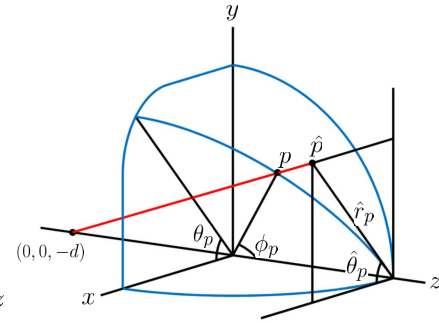

(b) The second step
Figure 2: The two-step projection model.

\section{The proposed projection model}

This section describes our projection model for mapping a point $\bar{p}$ on the viewing sphere to a point $\hat{p}$ on the image plane. For a full viewing sphere, we divide it into two hemispheres by the $x y$ plane and handle them separately. We start by describing the coordinate systems as illustrated in Figure 2. The $x z$ plane is parallel to the ground plane. The viewing sphere is centered at the origin and its radius is 1 . For projection, the viewing direction is towards the positive $z$ axis and the projection plane is located at $z=1$. A point $\bar{p}$ on the viewing sphere can be described by a $3 \mathrm{D}$ spherical coordinate $\left(r, \theta_{p}, \phi_{p}\right)$, where $r=1$ for the unit viewing sphere, $\phi_{p}$ is the angle measured from positive $z$ axis and $\theta_{p}$ is the angle measured on the $x y$ plane from the positive $x$ axis. The projected point $\hat{p}$ on the image plane can be represented by a $2 \mathrm{D}$ polar coordinate $\left(\hat{\theta}_{p}, \hat{r}_{p}\right)$.

\subsection{The two-step projection model}

Our projection model is a two-step model that maps from a hemisphere to the projection plane as illustrated in Figure 2 , which only shows an octant. Given a point $\bar{p}$ on the hemisphere (the orange surface), the first step projects $\bar{p}$ onto a point $p$ on a surface $S$ (the blue surface) through a line emanating from the center of the hemisphere. The surface can be formed by arbitrary spherical function that maps $(\theta, \phi)$ to a real value. By construction, the $3 \mathrm{D}$ spherical coordinate of $p$ is $\left(r_{p}, \theta_{p}, \phi_{p}\right)$ where its polar and azimuth angles are the same as $\bar{p}$ while the radial distance changes to $r_{p}$ depending on the definition of the surface $S$. 


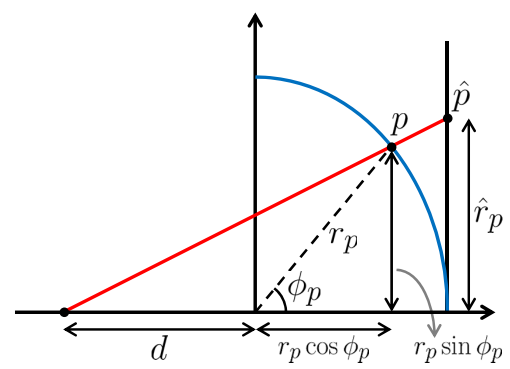

Figure 3: By applying similar triangles, we can obtain the formula for $\hat{r}_{p}$ (Equation 2).

In the second step, $p$ on the surface $S$ is projected onto a point $\hat{p}$ on the image plane by the perspective projection. As shown in Figure 2(b), we let the center of projection lie on the negative $z$ axis with coordinate $(0,0,-d)$. Since all projected points lie on the image plane, we can denote them using a $2 \mathrm{D}$ poplar coordinate system on the image plane. The 2D polar coordinate of $\hat{p},\left(\hat{\theta}_{p}, \hat{r}_{p}\right)$, can be expressed as

$$
\begin{aligned}
& \hat{\theta}_{p}=\theta_{p}, \\
& \hat{r}_{p}=\frac{d+1}{d+r_{p} \cos \left(\phi_{p}\right)} \cdot r_{p} \sin \phi_{p} .
\end{aligned}
$$

The formula of $\hat{r}_{p}$ can be obtained by similar triangles as shown in Figure 3. Note that $r_{p}$ and $\hat{r}_{p}$ are for different coordinate systems. $r_{p}$ denotes the radial distance in the 3D spherical coordinate system while $\hat{r}_{p}$ is for the 2D polar coordinate system. With Equation 1 and 2, one can relate the 3D spherical coordinate $\left(1, \theta_{p}, \phi_{p}\right)$ of a point $\bar{p}$ on the hemisphere with its projection $\hat{p}$ on the image plane with 2D polar coordinate $\left(\hat{r}_{p}, \hat{\theta}_{p}\right)$. Note that the calculation of $r_{p}$ depends on the surface $S$.

\subsection{Swung surfaces}

Our construction of the surface $S$ comes from an extension to the surface of revolution. Constructing a surface of revolution requires a profile curve $\gamma$ and a revolution axis. The profile curve is a plane curve. Let $A_{\gamma}$ denote the plane that $\gamma$ lies on. The revolution axis is required to lie on $A_{\gamma}$. By rotating the profile curve about the revolution axis, one can generate a surface of revolution. Swung surfaces are extensions to surfaces of revolution. In addition to the profile curve and the revolution axis, a trajectory curve $R$ is required for swung surfaces. $R$ must be a closed plane curve. Let $A_{R}$ denote the plane that $R$ lies on. $A_{R}$ is required to be perpendicular to the revolution axis. Therefore, $A_{R}$ is also perpendicular to $A_{\gamma}$. A swung surface is generated as follows. Similar to surfaces of revolution, the profile curve rotates about the revolution axis. But this time, when rotating, the profile curve is also scaled at the same time. The scaling is guided by the trajectory curve $R$. More specifically, the profile curve $\gamma$ is scaled along the line of intersection between the rotated $A_{\gamma}$ and the $A_{R}$, such that the rotated $\gamma$ intersects with the trajectory curve $R$.

In this paper, we only explore a specific family of swung surfaces defined as follows. We set the revolution axis to be the $\mathrm{z}$ axis which is also the optical axis of the perspective projection in the second step of our projection model. The profile curve is defined as a unit half circle on the $x z$ plane (Figure 4(a)). The trajectory curve is defined on the xy plane and is in a rounded rectangular shape (Figure 4(b)), which can be parameterized by $\theta$ as $R(\theta)$. We will describe this trajectory curve in more detail in Section 3.3. Note that the discussion in this subsection applies to all trajectory curves parameterized as $R(\theta)$ but not limited to the rounded rectangular curve we chose.

Figure 4(c) demonstrates the construction of the swung surface $S$. The circular arc is rotated (the green arrow) and then scaled (the red arrow) to touch the trajectory curve. The direction vector of the scaling is $[\cos \theta, \sin \theta, 0]^{T}$. A scaled circular arc becomes an elliptical arc by the construction process. The two axes of the elliptical arc are the scaling direction and the $\mathrm{z}$ axis respectively. Note that no matter how the circular arc is rotated and scaled, the resulting elliptical arc always passes through the point $(0,0,1)^{1}$.

Remember that, for computing Equation 2, given a point $\bar{p}=\left(1, \theta_{p}, \phi_{p}\right)$ on the hemisphere, we need to find the radial distance $r_{p}$ of its projection $p$ on $S$. For our swung surface, $p$ locates on the elliptical arc generated by rotating a unit circular arc $(\gamma)$ by $\theta_{p}$ and then scaled by $R\left(\theta_{p}\right)$. As shown in Figure 4(d), the two semi-axes of the ellipse are $R\left(\theta_{p}\right)$ and 1 (along $\left[\cos \theta_{p}, \sin \theta_{p}, 0\right]^{T}$ and the $\mathrm{z}$ axis) respectively, and $r_{p}$ can be obtained by

$$
r_{p}=\sqrt{R\left(\theta_{p}\right)^{2} \sin ^{2} \alpha_{p}+\cos ^{2} \alpha_{p}},
$$

where $\alpha_{p}$ is the parameter of the ellipse. That is, points on the ellipse can be represented as $\left(\cos \alpha_{p}, a \sin \alpha_{p}\right)$ where $a=R\left(\theta_{p}\right)$ is the aspect ratio of the ellipse. $\alpha_{p}$ and $\phi_{p}$ are related by

$$
\tan \alpha_{p}=R\left(\theta_{p}\right)^{-1} \tan \phi_{p} .
$$

By looking into Figure 3 and 4(d), Equation 2 becomes

$$
\hat{r}_{p}=\frac{d+1}{d+\cos \alpha_{p}} \cdot R\left(\theta_{p}\right) \sin \alpha_{p}
$$

Thus, once defining the trajectory by specifying $R(\theta)$, given a point $\bar{p}$ on a hemisphere with the polar and azimuthal angles $\theta_{p}$ and, $\phi_{p}$, one can find its projection's polar coordinate $\left(\hat{\theta}_{p}, \hat{r}_{p}\right)$ on the image plane using Equation 1 and 5.

\subsection{The trajectory curve}

Figure 5 shows several examples of surfaces constructed from different trajectory curves when using a unit half circle

\footnotetext{
${ }^{1}$ The elliptical arc also always passes $(0,0,-1)$, but we only focus on the half with positive $\mathrm{z}$ coordinates for projecting a hemisphere.
} 


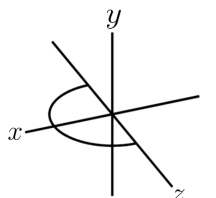

(a) Profile curve

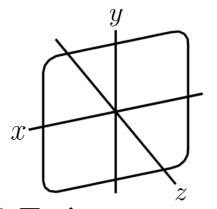

(b) Trajectory curve

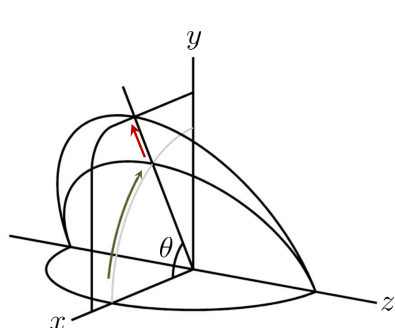

(c) Surface construction

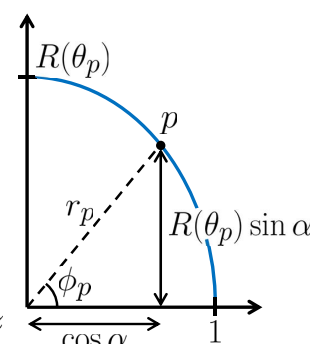

(d) Elliptical arc

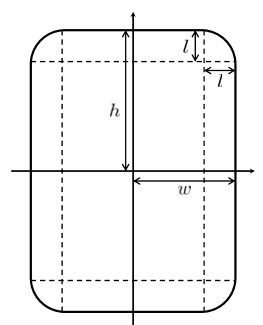

(e) Rounded rectangle

Figure 4: The construction of the swung surface $S$.

Circle Two vertical line
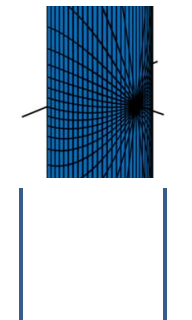

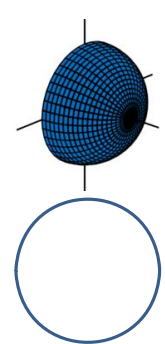

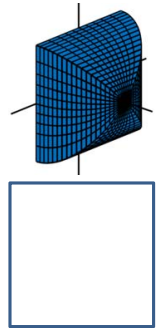

Square

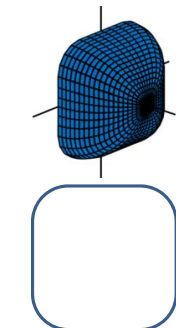

Rounded square
Figure 5: Examples of trajectory curves and the corresponding surfaces.

as the profile. For example, when using a circle $(R(\theta)=1)$ as the trajectory, the constructed surface is a sphere and the projection model becomes stereographic projection. If two vertical lines are employed as the trajectory, we obtain a cylinder as the surface and the model becomes Pannini projection. In this paper, we adopt a specific family of closed curves as the trajectory curve: rounded rectangles. Figure 5(d) shows the swung surface constructed using a rounded rectangle as the trajectory curve. It can be treated as an interpolation between the surfaces constructed by using the circle and the square as trajectories.

As shown in Figure 4(e), an rounded rectangle is parameterized by three parameters: width $(w)$, height $(h)$, and the radius of the circle $(l)$ for rounding corners. The center of the rounded rectangle coincides with the origin of the image plane. Since the image plane is located at the plane $z=1$, to maximize the conformality, we let $w=1$ and only vary $h$ which specifies the aspect ratio of the rectangle. Therefore, there are three parameters in our projection model: the focal length $d$, the aspect ratio $h$ and the roundness $l$. The later two determine $R(\theta)$ for the trajectory curve. When $l=0$, the curve becomes a rectangle. When $h=l=1$, it becomes an circle. Figure 6 visualizes impacts of the focal length and roundness. We usually prefer rectangular images. It can be achieved by setting lower roundness values. However, in such a configuration, a long line could take a sharp turn when running across the diagonals of the rectangle. As for $d$, a larger value of $d$ emphasizes more on the

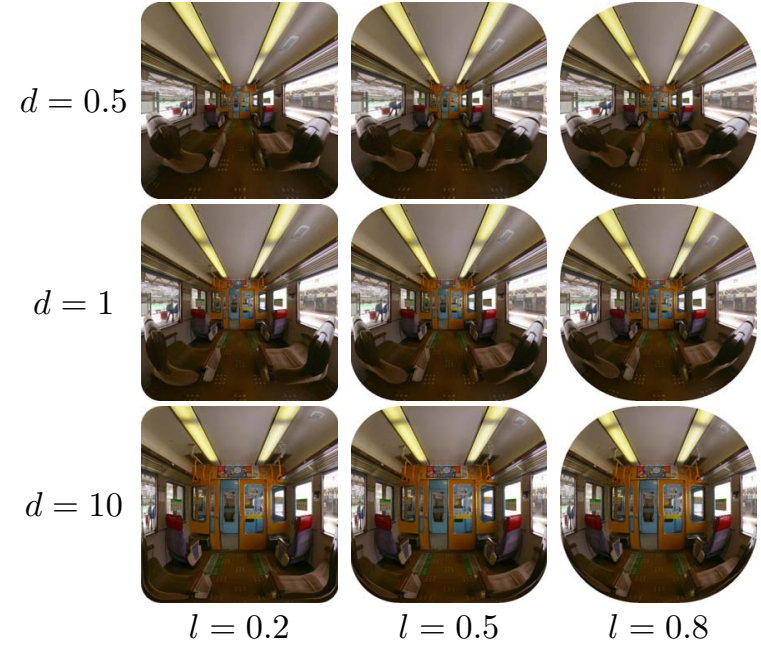

Figure 6: Visualizations with different parameters. In this example, the configuration of $d=1$ and $l=0.5$ or $l=0.8$ gives more visually pleasing results.

center part and squeezes the periphery. On the other hand, a smaller $d$ shrinks the center part and leaves more space for the periphery.

We adopt the round rectangles for several reasons. First, it is easy to control and optimize the shape of the rounded rectangle because of a small number of parameters. Second, the model is flexible enough and can be specialized to several well-known projections. Third, the shape of the projected image is axial symmetric. Thus, there is no need for cropping. The last but the most important reason is that four line segments of the rounded rectangle better preserve lines. We will have more discussions on this in Section 3.4.

\subsection{Comparisons with previous models}

Our projection model unifies the following projection models: rectilinear projection, stereographic projection, orthographic projection, Zorin and Barr's one-parameter family of projections that includes retilinear and stereographic projections, Ying and Hu's unified imaging model for catadioptric and fisheye cameras, and finally, the Pannini pro- 


\begin{tabular}{|l|c|c|}
\hline & $d$ & $R(\theta)$ \\
\hline Rectilinear & 0 & n/a \\
Stereographic & 1 & 1 \\
Orthographic & $\infty$ & 1 \\
Zorin [11] & {$[0,1]$} & 1 \\
Ying [9] & {$[0, \infty]$} & 1 \\
Pannini [6] & {$[0, \infty]$} & $\sqrt{1+\tan ^{2}(\theta)}$ \\
Ours & {$[0, \infty]$} & rounded rectangle \\
\hline
\end{tabular}

Table 1: Summary of different projection models. Our projection model unifies these projections.

jection. Table 1 shows the corresponding parameters $d$ and $R(\theta)$ for these projections. Note that, when $h$ in our model approaches to $\infty$, our model reduces to Pannini projection.

We used Tissot's indicatrix [5] to compare these projection models on distortions. Tissot's indicatrix is widely used in Cartography for characterizing distortions of map projections. The first column of Figure 7 shows the Tissot's indicatrix of rectilinear projection, stereographic projection, Pannini projection and the proposed model with a rounded square. It shows how a circle on the viewing sphere is mapped in the projection. Since stereographic projection is conformal, circles on sphere remain circular on the projection plane. For Pannini projection, circles near the zenith and nadir are severely distorted as ellipses. The distortion is the worst in rectilinear projection.

We then compare these methods on line preserving using grid patterns as shown in the middle column of Figure 7. Both vertical and horizontal lines becomes curved in stereographic projection. Pannini projection performs well on vertical lines but poorly on horizontal lines. Rectilinear projection works well on both. Our model is only second to rectilinear projection in terms of line preserving.

Figure 8(a) gives an example on why a vertical scene line keeps straight under the projection with a rounded rectangle. A vertical scene line is represented by a circular arc which passes through both the zenith and nadir (the orange arc). In the first step, when projecting onto the surface, it becomes a straight line (the blue line segment) because its nearby geometry is a elliptical cylinder (the blueshaded surface). In the second step, the blue line remains straight since perspective projection preserves lines. Thus, the straight line segments in the rounded rectangular trajectory produce a surface that is a portion of an elliptical cylinder which better preserves lines. If a scene line is parallel to the straight line segment of the trajectory, and its corresponding arc on the sphere (orange arc) lies in the orangeshaded surface, then the straightness of the scene line is preserved after the projection.

From the above discussion, we found that (1) stereographic projection performs better on distortion minimization because it is conformal. Pannini projection can preserve vertical scene lines because its trajectory curve con- (a)

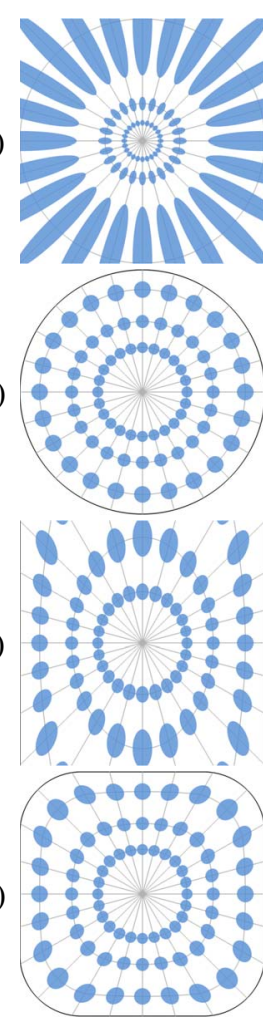

Tissot's indicatrix

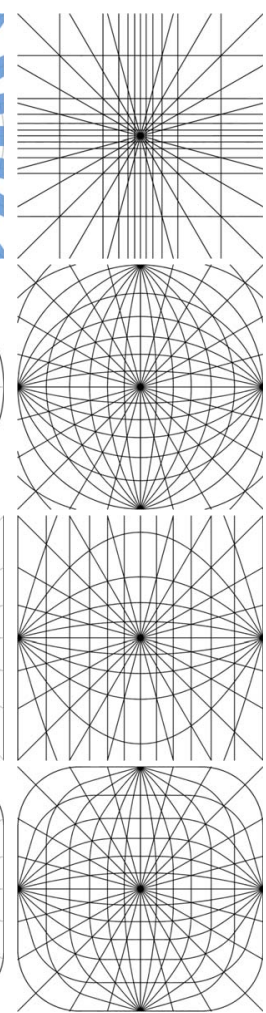

Grid pattern

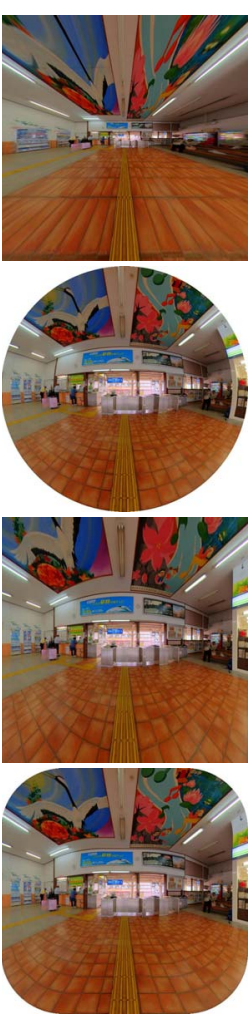

Projection
Figure 7: Tissot's indicatrix and grid patterns. (a) Rectilinear. (b) Stereographic. (c) Pannini. (d) Ours (rounded square). The FOV of rectilinear in this example is set to $160^{\circ}$. The FOV of the others are $180^{\circ}$. The result of Pannini is cropped in the vertical direction. The first column shows the Tissot's indicatrix. The grey lines are contours of either constant $\theta$ or constant $\phi$. The second column shows the projection of three sets of orthogonal scene lines.

tains vertical lines. Our projection model softly blends the advantages of stereographic projection and Pannini projection by a rounded rectangle composing both straight line segments and circular arcs. In addition, compared to Pannini projection, it contains horizontal line segments in the trajectory and can preserve horizontal scene lines in addition to vertical lines. Thus, our model strikes a better balance between distortion minimization and line preserving than previous models.

\subsection{Parameter setting}

The proposed projection model only has a handful of parameters and can be computed very efficiently, so it is not difficult for users to set parameters manually. Nevertheless, we provide users with an option for automatically setting parameters based on the image content. For the focal length, we set $d=1$ to maximize the conformality for reducing 


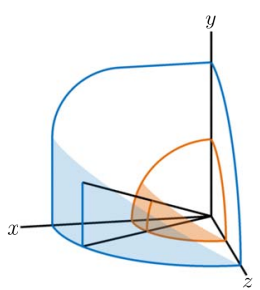

(a)

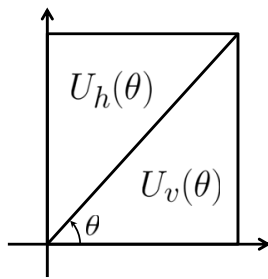

(b) aspect ratio

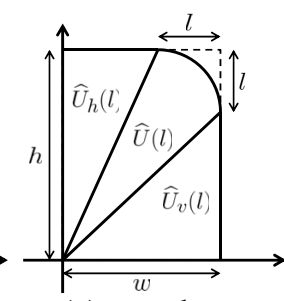

(c) roundness
Figure 8: (a) An example on how the proposed model better preserves lines. (b)(c) Definitions of areas for finding the best parameters for the proposed model.

content distortions. For the trajectory curve, we first find a good aspect ratio of the rectangle with zero roundness and then look for the best roundness of the rounded rectangles with that aspect ratio.

The content feature we used for optimizing the visual experience is lines. It is because human is often more sensitive to distortions of geometric structures, especially straight lines. Distortions of lines often look more noticeable and disturbing. For finding line structures, we used a cube map to project the viewing sphere onto six perspective views, then used the LSD line segment detector [7] to find line structures. Each line segment corresponds to an arc $s_{i}$ of a great circle on the viewing sphere.

Since the rectangle is axial symmetric to the $\mathrm{x}$ and $\mathrm{y}$ axes, we only explain the computation for one quadrant, and the same idea can be applied to other quadrants. The rectangular region in the first quadrant can be separated into two subregions $U_{v}(\theta)$ and $U_{h}(\theta)$ by a line with angle of inclination $\theta$ as shown in Figure 8(b). The angle of inclination $\theta$ is related with the aspect ratio $h$ by $h=\tan \theta$. From the previous analysis, we know that vertical (horizontal) scene lines in subregion $U_{v}(\theta)\left(U_{h}(\theta)\right)$ remain straight. On the other hand, vertical lines located in $U_{h}(\theta)$ will be severely distorted and the same for horizontal lines and $U_{v}(\theta)$. Thus, we would like to minimize the number of vertical/horizontal lines lying in $U_{h}(\theta) / U_{v}(\theta)$ through adjusting the aspect ratio. In addition, the aspect ratio deviating more from 1 will introduce more content distortions. Thus, we prefer the aspect ratio closer to 1 and use this criterion as the regularization term. We formulate these criteria as the following cost function for the aspect ratio,

$$
\begin{aligned}
E(\theta) & =\sum_{s_{i} \in S_{v}} \mathbb{1}\left[s_{i} \cap U_{h}(\theta) \neq \emptyset\right] \\
& +w_{h} \sum_{s_{i} \in S_{h}} \mathbb{1}\left[s_{i} \cap U_{v}(\theta) \neq \emptyset\right] \\
& +\lambda|\theta-(\pi / 4)|,
\end{aligned}
$$

where $\mathbb{1}[]$ is the indicator function; and $S_{v}$ and $S_{h}$ are the sets of vertical and horizontal scene lines respectively. The

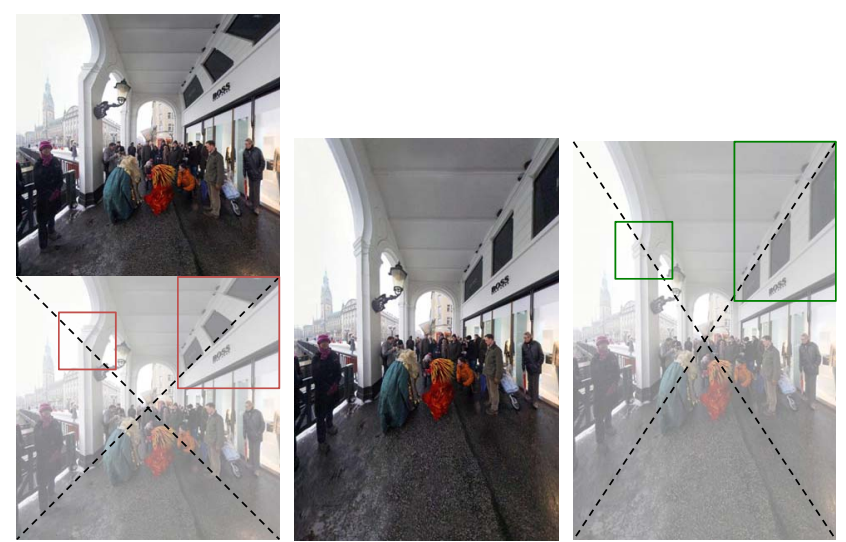

Figure 9: Comparisons of square and rectangle trajectories.

first term counts the number of vertical scene lines lying in the subregion $U_{h}$, and the second term counts horizontal lines for $U_{v}$. The third term is for regularization that prefers $\theta$ close to $\pi / 4$. By minimizing Equation 6 , we can find the best aspect ratio which better avoids line distortions. We used $w_{h}=0.4$ and $\lambda=40$ in our experiments. As there are four quadrants, the cost is calculated at each quadrant and then aggregated by taking the maximum operator. Finally, the optimization is performed by uniformly sampling the interval $[0, \pi / 2]$ with 90 samples, evaluating the cost function for the sampled values and picking up the one with the lowest cost.

After determining the aspect ratio, we would like to find the best roundness for the fixed aspect ratio. As show in Fig. 8(c), depending on $l$, the quadrant is divided into three region: $\widehat{U}_{h}(l), \widehat{U}_{v}(l)$ and the rounding area $\widehat{U}(l)$. The criteria are quite similar to the previous step and we have the following energy function,

$$
\begin{aligned}
\widehat{E}(l) & =\sum_{i \in S_{v}} \mathbb{1}\left[s_{i} \cap \widehat{U}_{h}(l) \neq \emptyset\right] \\
& +\widehat{w}_{h} \sum_{i \in S_{h}} \mathbb{1}\left[s_{i} \cap \widehat{U}_{v}(l) \neq \emptyset\right] \\
& +\widehat{\lambda} \cdot l
\end{aligned}
$$

The first two terms are similar to those in Equation 6. For example, we want that vertical scene lines lie in either $\widehat{U}_{v}(l)$ or $\widehat{U}(l)$, but not $\widehat{U}_{h}(l)$. Although a larger roundness could reduce sharp turns of lines and have less distortion, users usually prefer rectangle images. Thus, the third term prefers small roundness values. We used $\widehat{w}_{h}=1$ and $\widehat{\lambda}=24$ in our experiments.

\section{Experiments}

We implemented our method on a PC with a $3.4 \mathrm{GHz}$ CPU and 4GB RAM. For an output image with the $800 \times$ 

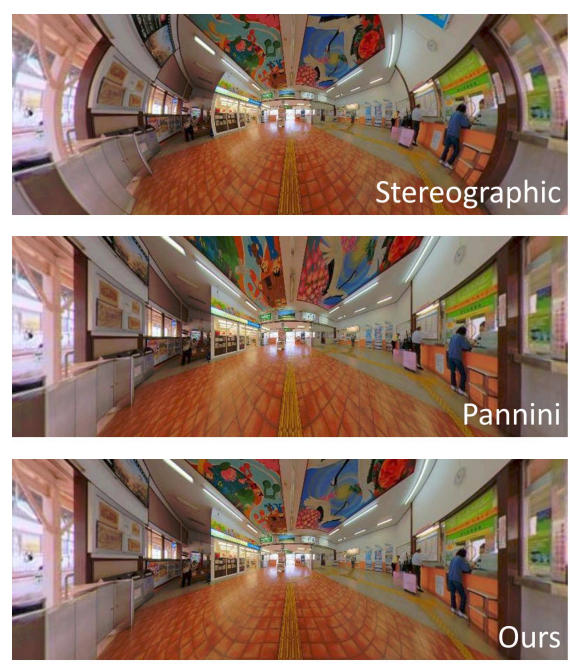

Figure 10: When the horizontal FOV is very wide, the line distortion of stereographic projection aggravates quickly. Pannini and ours achieve similar results in line preserving but ours have a larger vertical FOV.

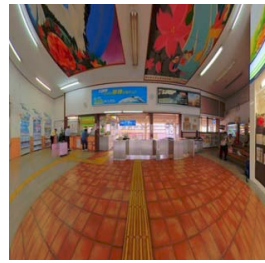

(a)

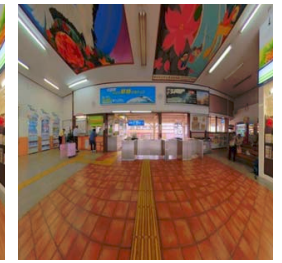

(b)

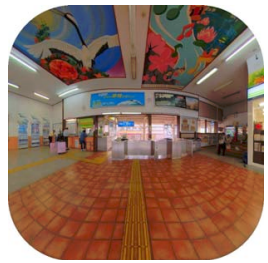

(c)
Figure 11: Comparisons with general Pannini projection (Pannini projection followed by vertical compression). (a) Hard compression. (b) Soft compression. (c) Ours.

800 resolution, our MATLAB implementation took around 1 second to find the parameters of projection, and less than 1 second for projection. The inputs were collected from Flickr and stored in the equirectangular format.

Figure 1 compares our model with previous models. In Figure 9, we show an example that the rectangular trajectory could further reduce distortions. In particular, discontinuity on the diagonal can be reduced by choosing a proper aspect ratio of the rectangular trajectory. Discontinuity occurs along dash lines representing the diagonals. With square trajectory, the pillar on the left suffers from a sharp turn of a vertical line. By finding a proper aspect ratio, one could hide the joint at a less obvious location. Figure 10 shows an example with FOV beyond a hemisphere (vertical 220 degrees and horizontal 300 degrees).

Figure 11 compares our projection with the general Pannini projection [6], which is a two-stage process: the Pannini projection followed by vertical compression (VC). VC is a mapping from $\mathbb{R}^{2}$ to $\mathbb{R}^{2}$ for straightening horizontal lines, with two options: hard compression (Figure 11(a)) and soft compression (Figure 11(b)). To compare our model with general Pannini projection more intuitively, one could interpret our model as a two-stage process: the Pannini projection followed by a warping from Panninis result (Figure $7(\mathrm{c})$ ) to our result (Figure 11(c)). The warping is achieved by changing parameters $(\theta$ and $l)$ and performing the two-step projection. Informally speaking, similar to general Pannini which is Pannini+VC, one could treat our model as Pannini+warping. They differ in the following way. VC refines the projected image (Figure 7(c)) by vertically stretching the image (although its name suggests compression). By doing so, some horizontal lines can be straightened while keeping vertical lines straight. However, it introduces distortions at both sides of the image and bends vanishing lines (Figure 11(a)(b)). Our model warps Figure 7(c) to Figure 11(c) by vertically squeezing the projected image (the actual process is more complicated). It reduces shape distortions and expands the vertical FOV. Although some vertical lines become curved, all radial lines are kept straight (as shown in the grid pattern of Figure 7(d)).

Figure 12 compares our method with content-preserving projection [2] which requires user-drawn lines. In terms of line preservation, our model is similar to Carroll et al.'s method. However, the resulting boundary of Carroll et al.'s method varies among images and usually is not symmetric. Thus, cropping is needed and information near the boundary could be lost. Our method does not suffer from the problem wirh cropping.

Our method can also be used for viewing full spherical panoramas or creating thumbnails for them. The full viewing sphere is cut into two hemispheres. To enhance the perspective perception, we find the cut so that the vanishing points coincide with the center of the hemispheres. With our model, the vanishing lines passing through vanishing points will be kept straight. Both hemispheres are processed independently. However, to make a nice symmetry between two hemisphere, the projection parameters were found jointly. Figure 13 shows the results and comparisons. Equirectangular projection severely distorts the content near the zenith and nadir. It also reduces the perspective perception as it bends the vanishing lines. Pannini projection is less effective in the vertical FOV. Stereographic projection bends lines. Our model is more effective compared to them.

\section{Conclusion}

This paper proposes a new projection model for visualizing wide-angle images. It unifies several previous models and shows better performance. Our current method only explores a subset of the general two-step projection. We would like to further explore the model (e.g. different profile curves) in the future. 


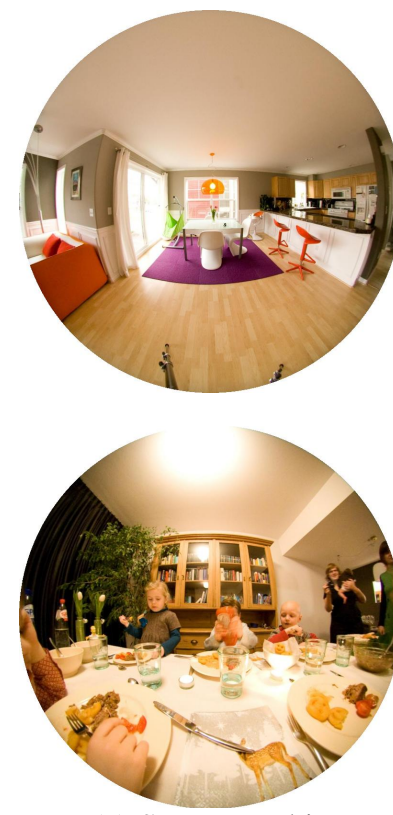

(a) Stereographic
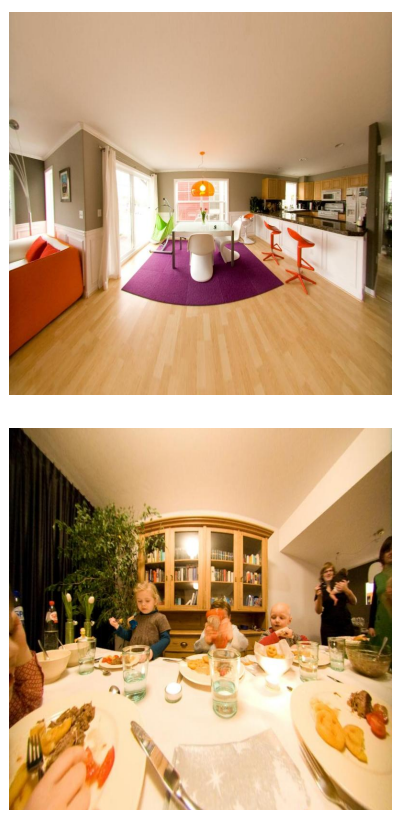

(b) Pannini

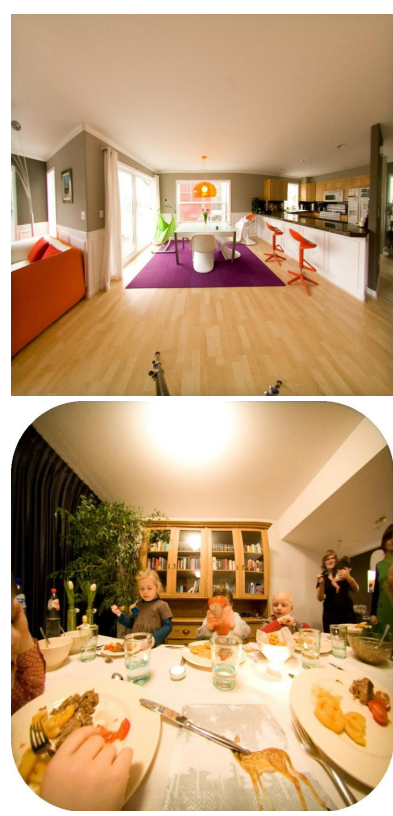

(c) Ours
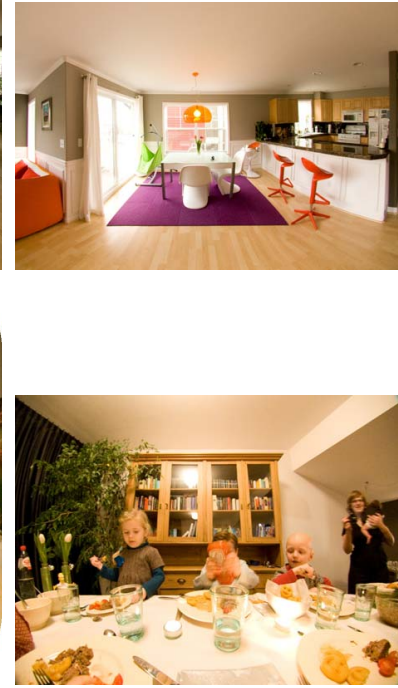

(d) Carroll et al. [2]

Figure 12: Comparisons with stereographic projection, Pannini projection and content-preserving projection.

(a)

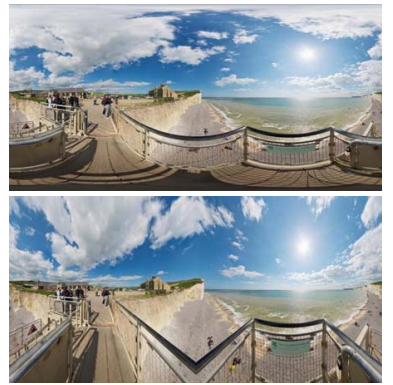

(c)

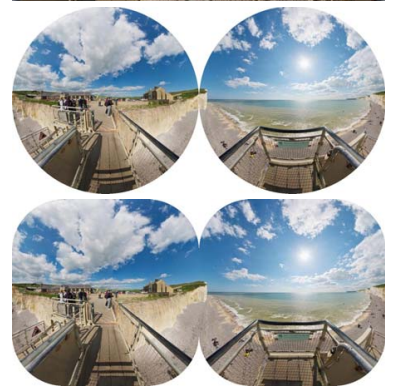

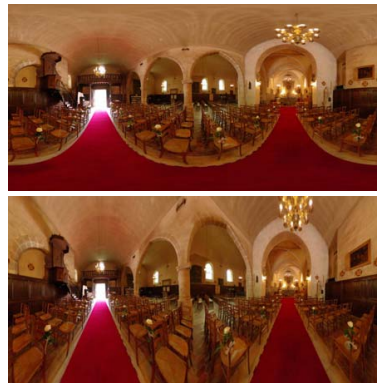

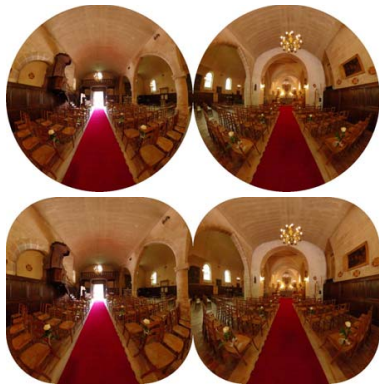

Figure 13: Full spherical panorama visualizations. Equirectangular projection. (b) Pannini projection. (a) Stereographic projection. (d) Our projection.

\section{References}

[1] A. Agarwala, M. Agrawala, M. Cohen, D. Salesin, and R. Szeliski. Photographing long scenes with multi-viewpoint panoramas. In ACM Transactions on Graphics, volume 25, pages 853-861, 2006. 2

[2] R. Carroll, M. Agrawal, and A. Agarwala. Optimizing

content-preserving projections for wide-angle images. In ACM Transactions on Graphics, volume 28, page 43, 2009. $2,7,8$

[3] J. Kopf, B. Chen, R. Szeliski, and M. Cohen. Street slide: browsing street level imagery. ACM Transactions on Graphics, 29(4):96, 2010. 2

[4] J. Kopf, M. Uyttendaele, O. Deussen, and M. F. Cohen. Capturing and viewing gigapixel images. ACM Transactions on Graphics, 26(3):93, 2007. 2

[5] P. H. Laskowski. The traditional and modern look at tissot's indicatrix. The American Cartographer, 16(2), 1989. 5

[6] T. K. Sharpless, B. Postle, and D. M. German. Pannini: a new projection for rendering wide angle perspective images. In Proceedings of the Sixth international conference on Computational Aesthetics in Graphics, Visualization and Imaging, pages 9-16, 2010. 2, 5, 7

[7] R. G. Von Gioi, J. Jakubowicz, J.-M. Morel, and G. Randall. LSD: A fast line segment detector with a false detection control. IEEE PAMI, 32(4):722-732, 2010. 6

[8] J. Wei, C.-F. Li, S.-M. Hu, R. R. Martin, and C.-L. Tai. Fisheye video correction. IEEE TVCG, 18(10):1771-1783, 2012. 2

[9] X. Ying and Z. Hu. Can we consider central catadioptric cameras and fisheye cameras within a unified imaging model. In Proceedings of ECCV 2004, pages 442-455, 2004. 2, 5

[10] L. Zelnik-Manor, G. Peters, and P. Perona. Squaring the circle in panoramas. In Proceedings of ICCV 2005, volume 2, pages 1292-1299, 2005. 2

[11] D. Zorin and A. H. Barr. Correction of geometric perceptual distortions in pictures. In Proceedings of ACM SIGGRAPH, pages $257-264,1995.2,5$ 